ORIGINAL ARTICLE

\title{
Crisis management during anaesthesia: myocardial ischaemia and infarction
}

\author{
G L Ludbrook, R K Webb, M Currie, L M Watterson
}

Qual Saf Health Care 2005;14:e13 (http://www.qshc.com/cgi/content/full/14/3/e13). doi: 10.1136/qshc.2002.004416

See end of article for authors' affiliations

Correspondence to: Professor W B Runciman, President, Australian Patient Safety Foundation GPO Box 400, Adelaide, South Australia 5001, Australia; research@apsf. net.au

Accepted 11 January 2005
Background: Myocardial ischaemia and infarction are significant perioperative complications which are associated with poor patient outcome. Anaesthetic practice should therefore focus, particularly in the at risk patient, on their prevention, their accurate detection, on the identification of precipitating factors, and on rapid effective management.

Objectives: To examine the role of a previously described core algorithm "COVER ABCD-A SWIFT CHECK" supplemented by a specific sub-algorithm for myocardial ischaemia and infarction in the management of myocardial ischaemia and/or infarction occurring in association with anaesthesia.

Methods: The potential performance of this structured approach for each of the relevant incidents among the first 4000 reported to the Australian Incident Monitoring Study (AIMS) was compared with the actual management as reported by the anaesthetists involved.

Results: Of the 125 incidents retrieved from the 4000 reports, 40 (1\%) were considered to demonstrate myocardial infarction or ischaemia. The use of the structured approach described in this paper would have led to appropriate management in $90 \%$ of cases, with the remaining $10 \%$ requiring other sub-algorithms. It was considered that the application of this structured approach would have led to earlier recognition and/ or better management of the problem in $45 \%$ of cases.

Conclusion: Close and continuous monitoring of patients at risk of myocardial ischaemia during anaesthesia is necessary, using optimal ECG lead configurations, but sensitivity of this monitoring is not $100 \%$. Coronary vasodilatation with glyceryl trinitrate (GTN) should not be withheld when indicated and the early use of beta blocking drugs should be considered even with normal blood pressures and heart rates.
M yocardial ischaemia and infarction are significant perioperative complications which are associated with poor patient outcome. ${ }^{12}$ Anaesthetic practice should therefore focus, particularly in the at risk patient, on their prevention, their accurate detection, on the identification of precipitating factors, and on rapid effective management. Because management of this problem by anaesthetists varies considerably, it was decided to examine the role of a structured approach.

In 1993 a "core" crisis management algorithm, represented by the mnemonic COVER ABCD-A SWIFT CHECK (the AB precedes COVER for the non-intubated patient), was proposed as the basis for a systematic approach to crisis management during anaesthesia where it is not immediately obvious what should be done or when actions taken have failed to remedy the situation. ${ }^{3}$ This was validated against the first 2000 incidents reported to the original Australian Incident Monitoring Study (AIMS). AIMS is an ongoing study which involves the voluntary anonymous reporting of any unintended incident which reduced or could have reduced the safety margin for a patient. ${ }^{4}$

It was concluded that, if this algorithm had been correctly applied, a functional diagnosis would have been reached in $40-60$ seconds in $99 \%$ of applicable incidents, and that the learned sequence of actions represented by the COVER portion would have led to appropriate steps being taken to handle the $60 \%$ of problems relevant to this portion of the algorithm. ${ }^{3}$ However, this study also showed that the $40 \%$ of problems represented by the remainder of the algorithm ABCD-A SWIFT CHECK were not always promptly diagnosed or appropriately managed..$^{3-5}$ It was decided that it would be useful, for these problems, to develop a set of sub-algorithms in an easy-to-use crisis management manual. ${ }^{6}$ This study reports on the potential place of the COVER ABCD-A SWIFT CHECK algorithm in the diagnosis and initial management of myocardial ischaemia, provides an outline of a specific crisis management algorithm for myocardial ischaemia and/or infarction during anaesthesia, and provides an indication of the potential value of using this structured approach.

\section{METHODS}

Of the first 4000 incidents reported to AIMS, those which made reference to "cardiac", "infarction", "infarct", "ischaemia", "myocardial", "ECG-changes" or "ECG-ischaemia" were extracted and analysed for relevance, presenting features, potential causes, diagnosis, management and outcome. The COVER ABCD-A SWIFT CHECK algorithm, described elsewhere in this series of articles, ${ }^{6}$ was applied to each relevant report to determine the stages at which the problem might have been diagnosed and to confirm that activating the COVER portion would have led to appropriate initial steps being taken. As myocardial ischaemia is not adequately dealt with by this algorithm, a specific subalgorithm for myocardial ischaemia and/or infarction was developed (see fig 1) and its putative effectiveness was tested against the reports. How this was done is described elsewhere in this series of articles. ${ }^{6}$ The potential value of this structured approach - that is, the application of COVER ABCD-A SWIFT CHECK to the diagnosis and initial management of the problem followed by the application of the myocardial ischaemia or infarction sub-algorithm-was assessed in the light of the AIMS reports by comparing its potential effectiveness for each incident with that of the actual management as recorded in each report. 


\section{MYOCARDIAL ISCHAEMIA}

\section{SIGNS $(1)^{*}$}

ST changes - elevation or depression

$\mathrm{T}$ wave flattening or inversion

Ventricular dysrhythmias

\section{PRECIPITATING FACTORS}

Pre-existing cardiovascular disease

Haemodynamic instability

Tachy- or bradycardia

Hyper or hypotension

Desaturation

Pulmonary oedema

Awareness/light anaesthesia/intubation (2)

\section{EMERGENCY MANAGEMENT}

Inform the surgeon

Defer, or rapidly complete, surgery

Ensure adequate oxygenation

Correct any haemodynamic derangement (3)

If hypotensive $\rightarrow$ page $32^{* *}$

If hypertensive $\rightarrow$ page $34^{* *}$

If tachycardic $\rightarrow$ page $30^{* *}$

If bradycardic $\rightarrow$ page $28^{* *}$

\section{If ischaemia does not resolve rapidly (4)}

Commence glyceryl trinitrate $(50 \mathrm{mg}$ in $500 \mathrm{ml} 5 \%$ dextrose) and start at $0.1 \mathrm{ml} / \mathrm{kg} / \mathrm{hr}$

Titrate against clinical response

Consider multilead ECG monitoring (5)

Monitor ECG continuously

Aim for haematocrit - $30 \%$

If the myocardial ischaemia is significant, consider short-acting

$\beta$-blocker to cover emergence.

The sub-algorithm forms a facing page of the

Crisis Management Manual16.

* Numbers in brackets refer to Notes in the right hand panel.

** Page references refer to Crisis Management Manual16.

\section{FURTHER MANAGEMENT}

Obtain a 12 lead unfiltered ECG as soon as possible to assist in the diagnosis.

Admit to HDU/ICU/CCU

Consider invasive monitoring:

Blood pressure

Cardiac filling pressures

Further investigation - serial ECG/cardiac enzymes

Continue oxygen therapy for at least 2 days.

\section{NOTES:}

It was judged that correct use of the algorithm would have led to appropriate management in $90 \%$ of cases, and would have led to earlier recognition of the problem and/or better management in $47 \%$ of the reported cases. The remaining $10 \%$ would have required the use of sub-algorithms (eg. air embolism).

(1) In all cases, ECG changes were reported as the means of detection. The diagnosis should be confirmed where possible by comparing monitor changes to a pre-operative trace.

In $73 \%$ of cases there were associated cardiorespiratory abnormalities:

$43 \%$ - hypotension;

$25 \%$ - tachycardia/hypertension; $5 \%$ - desaturation.

(2) $15 \%$ of cases were judged secondary to light anaesthesia and $50 \%$ of these occurred with intubation.

(3) Resolution of ischaemia followed correction of cardiorespiratory abnormalities alone in $35 \%$ of cases.

(4) In the incidents reported, it was considered that in $40 \%$ of cases coronary vasodilator treatment, or more rapid treatment was indicated.

(5) It is well recognised that Standard 3 lead monitoring for ischaemia is very insensitive. Use the CM5 configuration to maximise the detection of ischaemia if multilead monitoring is not in use.

These notes comprise a reverse side of a page of the Crisis Management Manual ${ }^{16}$.

Figure 1 Myocardial ischaemia.

\section{RESULTS}

Using the criteria described above, 125 incidents were retrieved from the first 4000 AIMS reports. After individual examination it was considered that myocardial infarction or ischaemia had occurred in 40 of these (that is, in $1 \%$ of incidents in the database). Myocardial infarction was considered to have occurred in eight incidents and myocardial ischaemia in 32. In all incidents electrocardiography (ECG) was reported as the means of detection. Transoesophageal echocardiography (TOE) was not used in any of the 125 incidents retrieved.

In 29 cases there were associated cardiorespiratory abnormalities of sufficient magnitude to warrant correction even if not accompanied by ischaemia (hypotension in 17 cases, tachycardia or hypertension in 10 , and desaturation in two). In 11 cases ECG changes were the only initial evidence of a problem.

Of the 17 incidents associated with hypotension, four followed rapid intravenous injection of drugs at induction, three were due to overdose of non-anaesthetic drugs, two were associated with embolism, one was associated with onset of a subarachnoid block, one was thought to be due to peritoneal traction, one was due to hypovolaemia secondary to blood loss, and the remaining five were cases of hypotension which occurred for no identifiable reason during maintenance of anaesthesia. Of this hypotensive group, ischaemia was corrected in six cases by the restoration of normotension alone and in four cases by restoration of normotension and additional treatment with glyceryl trinitrate $(\mathrm{GTN})$.

Of the 10 incidents associated with hypertension or tachycardia, six were secondary to light anaesthesia (three at intubation), one followed drug administration, and three occurred in theatre prior to induction. Three cases of ischaemia were corrected by a reduction in heart rate alone; six cases received GTN which corrected ischaemia in all but one.

Two cases of myocardial ischaemia followed severe desaturation. This was secondary to laryngospasm at induction in one case and was accompanied by tachycardia, while in the other hypoxia was secondary to airway obstruction. In one case ECG changes resolved once oxygenation was restored, but in the other ischaemia persisted.

Eleven of the incidents were not associated with clinically identifiable abnormal physiological parameters and were detected by ECG alone. These were termed "silent" ischaemia or infarction. Only one case received GTN which was successful in correcting the ischaemia. In five cases no active treatment was given and in two of these the ischaemia persisted for a prolonged period. In two incidents in this 
"silent" group ECG changes were detected before induction, yet in both cases anaesthesia was commenced without corrective action being taken.

When the COVER ABCD-A SWIFT CHECK algorithm was applied to each report, it was considered that the diagnosis of myocardial ischaemia or infarction would have been made at the Rl (Review monitors) stage of COVER in all cases. ${ }^{6}$ It was considered that reversal of associated cardiorespiratory changes alone, which would have been effectively diagnosed by use of the COVER ABCD portion of the algorithm, would have led to correction of ischaemia in 10 incidents (hypotension in six cases, tachycardia in three, and desaturation in one). Application of the ischaemia/infarction subalgorithm (fig 1) would have led to appropriate management in a further 26 incidents. It was therefore considered that application of COVER ABCD would theoretically have led to appropriate effective management in $25 \%$ of the 40 incidents of ischaemia or infarction reported, and that the addition of the ischaemia/infarction sub-algorithm would have led to appropriate management being used in $90 \%$ of cases. In four cases $(10 \%)$ it was considered that application of other specific sub-algorithms was indicated. These involved water intoxication secondary to bladder irrigation in one case and embolism in three. While concomitant treatment of ischaemia would probably not have been inappropriate in these cases, it was considered that application of other specific subalgorithms would have been required.

When the potential effectiveness of the structured approach represented by the COVER ABCD-A SWIFT CHECK algorithm and the special sub-algorithm for ischaemia or infarction was compared with that of the actual management as documented in each of the 40 incident reports, it was considered that, properly applied, the structured approach recommended would have been likely to have led to quicker and/or better resolution of the problem in 18 cases $(45 \%)$. In 16 of these cases it was considered that treatment, or more rapid treatment, with a coronary vasodilator was indicated.

\section{DISCUSSION}

Incidents of myocardial ischaemia or infarction retrieved from the AIMS database represented $1 \%$ of all reported incidents; however, many incidents involving ischaemia or infarction which manifested as other problems such as cardiac arrest or dysrhythmia would not have been retrieved with the criteria used for this search of the database. Furthermore, intraoperative ischaemia occurs much more frequently than is detected by monitors such as the ECG. All cases of ischaemia or infarction reported to AIMS were detected by ECG; there were no reports of detection by transoesophageal echocardiography, reflecting the low rate of usage of these monitors in Australia at the time the first 4000 incidents were reported.

It is now well recognised that even sophisticated ECG devices with automated segment analysis detect only a proportion of ischaemic events. ${ }^{78}$ Furthermore, electronic filtering, lead selection, the number of leads monitored, and only intermittent checking of the ECG trace may reduce this still further. ${ }^{18}$ Correct lead selection is particularly important and a full 12-lead ECG, although often impractical intraoperatively, remains the "gold standard" if accurate electrical diagnosis is required. For the high risk patient, intraoperative monitoring of leads $\mathrm{V}_{5}$ and $\mathrm{V}_{4}$ and II (in that order of priority) is likely to optimise the chances of ischaemia detection, but requires a more complex system than the usual 3 lead ECG in common use. ${ }^{9}{ }^{10}$ It must also be remembered that not all ST segment changes on ECG represent ischaemia, ${ }^{11}$ and some of the incidents reported here may not have been due to ischaemia. Finally, it must be remembered that the ECG may remain normal in the face of severe hypotension, arterial desaturation, hypercarbia and metabolic acidosis, and that it should not be relied upon, in any way, as a monitor of systemic abnormalities. ${ }^{52}$

Maintenance of the balance of myocardial oxygen supply and demand is widely recognised as fundamental to the prevention and treatment of ischaemia and infarction. ${ }^{13}$ An increased incidence of perioperative myocardial infarction in patients who had been hypotensive or tachycardiac intraoperatively provides evidence of the importance of precise control of heart rate and blood pressure in the "at risk" patient ${ }^{13}$ and the use of transoesophageal echocardiography in high risk patients. ${ }^{14}$ This is supported, for example, by the mounting evidence of the cardioprotective effects of perioperative beta blockers. ${ }^{15}$

Nearly three quarters of the cases of ischaemia or infarction in this series occurred in association with significant cardiorespiratory abnormalities which themselves would have warranted an incident report. Although cause and effect could not necessarily be differentiated, ischaemia was reversed by correction of these cardiorespiratory abnormalities alone in 10 of the 29 cases. It may be reasonable to assume, at least in these cases, that ischaemia was secondary to these abnormalities. Induction and intubation were obvious precipitants of cardiovascular instability associated with ischaemia or infarction in seven cases, emphasising the need for continuous on-line monitoring, appropriate dose regimens, and a rapid response to cardiovascular abnormalities, particularly in the at risk patient. Analysis of the relative effectiveness of the actual treatment instituted and COVER revealed that practising anaesthetists

\section{Key messages}

- Forty (1\%) of the first 4000 incidents reported to AIMS were considered to demonstrate myocardial infarction $(n=8)$ or ischaemia $(n=32)$.

- In all 40 incidents electrocardiography (ECG) was reported as the means of detection. Transoesophageal echocardiography was not used in any of the cases.

- Twenty nine cases (73\%) were associated with significant cardiorespiratory abnormalities: 17 with hypotension and 10 with hypertension or tachycardia.

- Eleven cases not associated with any clinically identifiable abnormal physiological parameters ("silent" ischaemia or infarction) were detected by ECG alone. This emphasises the importance of continuous cardiac monitoring.

- Acknowledging the low sensitivity of even sophisticated ECGs for detecting ischaemic events, intraoperative monitoring of leads $V_{5}$ and $V_{4}$ and lead II, in descending order of priority, offers optimal conditions.

- In this series anaesthetists did not perform well with the institution of coronary vasodilator therapy (such as GTN) when it seemed to be indicated.

- Follow up in recovery with a 12-lead ECG, serial ECG, cardiac enzymes and cardiological consultation are important in these patients.

- It was considered that the structured approach using both the "core" algorithm and then the specific subalgorithm for myocardial ischaemia or infarction, properly applied, would have been likely to have led to a quicker and/or better resolution of the problem in $18(45 \%)$ of the 40 cases. 
performed well in the diagnosis and management of underlying cardiorespiratory problems, with early appreciation of the significance of these abnormalities and rapid correction.

In the face of optimal cardiorespiratory parameters, myocardial perfusion may be improved with coronary vasodilators; GTN is the drug of first choice in this context in anaesthetic practice. It was the only vasodilator used to correct ischaemia in this series and proved effective, when used, in all but one case. The use of other coronary vasodilators such as calcium channel antagonists and adenosine may have been limited by lack of either availability or familiarity with dose regimens. It was considered that, in general, anaesthetists did not perform well with respect to the rapid institution of coronary vasodilator therapy when it seemed to be indicated, and that the use of a structured algorithm (fig 1) would have improved this aspect of management in $40 \%$ of cases. At the time the first 4000 incidents were reported, the use of beta blockers was limited. Early use of beta blockers should be considered, particularly with normal or high blood pressure and normal or high heart rates. ${ }^{14}{ }_{15}$

It is also important to note that, in one quarter of the cases of ischaemia/infarction in this series, there was no recognisable precipitating event, emphasising the need for continuous cardiac monitoring in patients deemed to have a significant risk of ischaemia or infarction, and the need for the selection and proper use of the most effective equipment.

The management of patients with acute myocardial infarction or evidence of ischaemia does not end with successful arrival in recovery. A 12-lead ECG should be obtained as soon as possible and compared with the preoperative ECG, if available. Serial ECG and cardiac enzymes should be considered in conjunction with a cardiologist, as should the ongoing management of the patient in a suitably equipped and staffed high dependency or intensive care unit. Finally, it is important that a full explanation of what happened be given to the patient and the problem clearly documented in the anaesthetic record. If a particular precipitating event was significant or a particular action was useful in resolving the crisis, this should be clearly explained and documented.

\section{ACKNOWLEDGEMENTS}

The authors would like to thank all the anaesthetists in Australia and New Zealand who contributed to the 4000 incident reports upon which this and the other 24 papers in the Crisis Management Series are based. The coordinators of the project also thank Liz Brown for preparing the draft of the original Crisis Management Manual; Loretta Smyth for typing; Monika Bullock RN for earlier coding and classifying of data; Dr Charles Bradfield for the electronic version of the algorithms; Dr Klee Benveniste for literature research; and Drs Klee Benveniste, Michal Kluger, John Williamson and Andrew Paix for editing and checking manuscripts.

\section{Authors' affiliations}

G L Ludbrook, Professor, Department of Anaesthesia and Intensive Care, University of Adelaide and Royal Adelaide Hospital, Adelaide, South Australia, Australia

R K Webb, Senior Staff Specialist, Department of Anaesthesia and Intensive Care, The Townsville Hospital, Douglas, Queensland, Australia M Currie, Clinical Quality Co-ordinator, Goulburn Base Hospital, Goulburn; and Consultant in Clinical Quality, Southern Area Health Service, New South Wales, Australia

L M Watterson, Senior Staff Specialist and Director, Sydney Medical Simulation Centre, Royal North Shore Hospital, St Leonards, New South Wales, Australia

This study was coordinated by the Australian Patient Safety Foundation, GPO Box 400, Adelaide, South Australia 5001, Australia.

\section{REFERENCES}

1 Edwards ND, Reilly CS. Detection of perioperative myocardial ischaemia Br J Anaesth 1994:72:104-15.

2 Fehr DM, Chambers CE, Thiele B, et al. Perioperative myocardial infarction in a patient undergoing abdominal aortic aneurysm resection: perioperative risk assessment. J Cardiothorac Vasc Anesth 1995;9:452-9.

3 Runciman WB, Webb RK, Klepper ID, et al. Crisis management: validation of an algorithm by analysis of 2000 incident reports. Anaesth Intensive Care 1993;21:579-92.

4 Webb RK, Currie M, Morgan CA, et al. The Australian Incident Monitoring Study: an analysis of 2000 incident reports. Anaesth Intensive Care 1993;21:520-8.

5 Webb RK, van der Walt JH, Runciman WB, et al. Which monitor? An analysis of 2000 incident reports. Anaesth Intensive Care 1993;21:529-42.

6 Runciman WB, Kluger MT, Morris RW, et al. Crisis management during anaesthesia: the development of an anaesthetic crisis management manual. Qual Saf Health Care 2005; 14:el.

7 Ellis JE, Shah MN, Briller JE, et al. A comparison of methods for the detection of myocardial ischaemia during non-cardiac surgery: automated ST-segment analysis systems, electrocardiography, and transesophageal echocardiography. Anesth Analg 1992;75:764-72.

8 Smith JS, Cahalan MK, Benefiel DJ, et al. Intraperative detection of myocardial ischemia in high-risk patients: electrocardiography versus two-dimensional transesophageal echocardiography. Circulation 1985;72:1015-21.

9 London MJ, Hollenberg M, Wong MG, et al. Intraoperative myocardial ischaemia: localisation by continuous 12-lead electrocardiography. Anesthesiology 1988;69:232-41.

10 Landesberg G, Mosseri M, Wolf $Y$, et al. Perioperative myocardial ischemia and infarction: identification by continuous 12-lead electrocardiogram with on-line ST-segment monitoring. Anesthesiology 2002;96:264-70.

11 Slogoff S, Keats AS, David Y, et al. Incidence of perioperative myocardial ischemia detected by different electrocardiographic systems. Anesthesiology 1990;73:1074-81.

12 Hall RI. Anaesthesia for coronary artery surgery-a plea for a goal-directed approach. Can J Anaesth 1993;40:1178-94.

13 Shah KB, Kleinman BS, Sami H, et al. Reevaluation of perioperative myocardial infarction in patients with prior myocardial infarction undergoing non-cardiac operations. Anesth Analg 1990;71:231-5.

14 Skidmore KL, London MJ. Myocardial ischemia. Monitoring to diagnose ischemia: how do I monitor therapy? Anesthesiol Clin North Am 2001; 19:651-72.

15 Auerbach AD, Goldman L. Beta-blockers and reduction of cardiac events in noncardiac surgery: scientific review. JAMA 2002;287:1435-44.

16 Australian Patient Safety Foundation. Crisis Management Manual: COVER $A B C D$ A SWIFT CHECK. Adelaide: Australian Patient Safety Foundation, 1996, 74 pp. Available at http://www.apsf.net.au/anaesthesia.htm (accessed 6 September 2004). 